\title{
Le morcellement informel du foncier en Algérie*
}

\author{
Faouzi Bouchaib ${ }^{1}$ \\ Anne-Marie Jouve ${ }^{2}$ \\ 1 Université Saad Dahlab \\ Département d'agronomie \\ Cité 168 logements (Bananiers) \\ Batiment 19, n⿳ 13 \\ 0009 Blida \\ Algérie \\ <bouchaib_fawzi2001@yahoo.fr> \\ ${ }^{2}$ Institut agronomique méditerranéen \\ de Montpellier \\ UMR MOISA \\ 3191 route de Mende \\ 34093 Montpellier cedex 5 \\ France \\ <jouve@iamm.fr>
}

\begin{abstract}
Résumé
La réforme agricole adoptée en Algérie en 1987 a décidé le partage des domaines agricoles socialistes (DAS) et la création des exploitations agricoles collectives (EAC) et exploitations agricoles individuelles (EAI), en laissant la propriété de la terre à l'État. En dépit des règles imposées par cette réforme - obligation du mode de production collectif aux EAC et interdiction du mode de faire valoir indirect - le morcellement informel des exploitations et le mode de faire-valoir indirect se sont généralisés très rapidement. L'enquête qualitative réalisée auprès d'un échantillon de 48 agriculteurs dans des EAC du périmètre irrigué de la Mitidja Ouest a montré que l'échec de la forme de production collective s'explique par les difficultés rencontrées par les attributaires à cause du passage brutal du système socialiste à une forme de gestion collective autonome qui aurait nécessité une préparation. Par la suite, l'évolution vers un morcellement informel des exploitations et le développement du mode de faire-valoir indirect, tous deux interdits par la loi, se sont traduits par une diversité comportementale des attributaires (dix types de comportements).
\end{abstract}

Mots clés : Algérie ; exploitation agricole collective ; socialisme ; typologie.

Thèmes : systèmes agraires ; territoire, foncier, politiques agricoles et alimentaires.

\section{Abstract \\ The informal divisions of collective farms in Algeria}

The agricultural reform adopted in Algeria in 1987 dictated the division of the Socialist Agricultural Domains and the creation of Collective and Individual Farms (EAC and EAI), the State keeping land ownership. In spite of the rules imposed by this reform, the obligation of the collective mode of production for the EAC and the banning of tenant farming, the informal division of the farms and farming by tenants have spread very quickly. The qualitative investigation carried out on a sample of 48 farmers in some EAC in the irrigated perimeter of Western Mitidja showed that the failure of the collective production form can be explained by the difficulties encountered by the beneficiaries because of the sudden change from the socialist system to a form of autonomous collective management for which preparation would have been required. Thereafter, the evolution towards an informal division of farms and the development of farming by tenants, both being illegal, resulted in behavioural diversity among the beneficiaries (ten types of behaviours).

Key words: Algeria; collective farms; socialism; typology.

Subjects: farming systems; territory, land use, agricultural and food production policy.

a décennie 1990 est marquée en Algérie, comme dans la plupart des pays socialistes, par un tournant libéral, caractérisé par la fin du collectivisme et du centralisme étatique. Mais la transition vers l'économie de marché emprunte des voies différentes dans chaque pays, notamment en ce qui concerne les droits de propriété (Jouve, 2009). L'Algérie, à l'instar de la Chine, 
mais contrairement aux pays de l'Europe de l'Est, a sauvegardé l'idéologie socialiste et maintenu la propriété étatique de la terre. Ainsi, la réforme agricole, adoptée en Algérie en 1987 (loi 87-19), qui a décidé le partage des domaines agricoles socialistes (DAS) et la création des exploitations agricoles collectives (EAC) et exploitations agricoles individuelles (EAI), n'a donné qu'un droit de jouissance de la terre aux nouveaux exploitants. De plus, dans le but de limiter le morcellement de la terre et la spéculation foncière, cette loi a introduit deux obligations: la première impose aux EAC le mode de production collectif et la seconde interdit le mode de faire-valoir indirect. Toutefois, en dépit de ces règles, un processus informel de partage des EAC et de recours au faire-valoir indirect s'est rapidement développé du fait de l'impréparation de la réforme et des difficultés rencontrées par les attributaires sur les plans humain, économique et financier. La prolifération de ces arrangements informels et illégaux n'empêche pas d'ailleurs un essor agraire non négligeable dans certains périmètres (Kuper et al., 2009).

L'étude de cas présentée ici cherche à analyser et à expliquer le morcellement informel des exploitations agricoles collectives et la diversité comportementale des attributaires dans le périmètre irrigué de la Mitidja Ouest. Dans un premier temps, il nous a paru indispensable de remonter à l'origine de ces processus et de faire un historique de la réforme de 1987.

\section{Historique : les origines du morcellement informel}

En 1963, les terres agricoles coloniales ont été étatisées et exploitées selon un modèle d'autogestion bicéphale inspiré de l'expérience yougoslave ; mais en réalité, ce modèle était très dirigiste et totalement sous le contrôle de l'État (Bédrani, 1981). Ces terres concernaient 2,5 millions d'hectares, le reste gardant un statut privé (Le Coz, 1991). Ces orientations socialistes et centralisées ont été maintenues jusqu'au milieu des années 1980, dans le contexte d'une réelle continuité politique, à travers les réformes agraires de 1971 et 1981. Cependant, malgré les correctifs apportés au fonctionnement des domaines socialistes, les performances agricoles restaient faibles et les déficits financiers très élevés. Ce constat d'échec conduisit à une semi-rupture d'orientation libérale (loi 87-19), visant à relancer le secteur privé et à moderniser l'agriculture grâce à des exploitations agricoles efficaces autonomes (Le Coz, 1991).

Sur le plan foncier, cette réforme a entraîné un morcellement de la terre par la création de 22356 exploitations agricoles collectives (EAC) et 5677 exploitations agricoles individuelles (EAI) à partir des ex-domaines agricoles socialistes (3 400 DAS), d'après le bilan fait en 1992 (Baci, 1999). Les attributaires proviennent essentiellement du personnel permanent des ex-DAS: gestionnaires, comptables, chefs de cultures, chefs d'étables, ouvriers, mécaniciens, etc. Toutefois d'autres ayants droit - anciens moudjabidines (combattants de la révolution), cadres de l'agriculture - ont pu également bénéficier d'une quotepart sur présentation d'un dossier. La quote-part foncière est en moyenne de 3 hectares en irrigué et de 6 hectares en sec.

Du point de vue organisationnel, la loi 87-19 a cédé le pouvoir de décision aux attributaires qui se sont organisés en groupes (d'au moins trois attributaires), formés généralement sur la base d'une affinité familiale, de voisinage ou amicale, et ont choisi un chef qui devait représenter le collectif et assurer la gestion de l'exploitation (souvent un ancien cadre du DAS). Cette loi constituait donc une véritable rupture qui permettait le désengagement de l'État, aussi bien sur le plan organisationnel qu'économique et financier. Cependant, la mise en place rapide de cette nouvelle forme de production collective a engendré des conflits internes entre les membres des EAC.

Dès les premières années, le partage des EAC (foncier et autres moyens de production), pourtant interdit par la loi, s'est présenté comme la solution ultime au rétablissement de l'ordre, avec un éventail très large de cheminements (Le Coz, 1991). En 2005, une enquête socioéconomique, réalisée sur 182 exploitations agricoles collectives, dans la commune de Mouzaïa dans la Mitidja, a montré que $73 \%$ des EAC sont "éclatées " avec un partage de facto de la terre entre attributaires (Imache, 2008).

On peut évoquer quelques causes de ce morcellement informel rapide et quasi général des EAC qui s'explique tantôt par le refus de l'autorité du chef du groupe par les autres membres, tantôt par l'incompétence du chef du groupe, tantôt par des divergences entre les membres du groupe sur la manière de gérer l'exploitation, tantôt par le manque de confiance ou par l'influence de l'environnement social. Cela s'est traduit par des conflits, de la désobéissance, de l'absentéisme, une préférence pour le travail individuel, etc. : autant de causes qui mettent en évidence la pertinence de l'aspect psychosociologique dans l'évolution socio-économique des exploitations agricoles collectives.

Pour comprendre cette évolution agricole et foncière dans le secteur agricole privé de l'État, nous avons cherché à analyser les comportements des attributaires des EAC. Notre hypothèse est la suivante : le morcellement informel a pour origine le passage trop brutal du système de production étatique à une nouvelle forme de production collective, autonome et responsable de sa gestion. En effet, les attributaires étaient en majorité des ouvriers et donc de simples exécutants dans les DAS, et le passage au statut de gestionnaire a été vécu difficilement. Par conséquent, la cause principale du morcellement des EAC réside vraisemblablement dans l'impréparation de la réforme de 1987. Par la suite, et après l'éclatement du pouvoir de décision économique, une diversité comportementale des attributaires s'est manifestée. Dans un contexte national encore instable et d'insécurité foncière liée aux transactions informelles, de nouveaux acteurs accèdent à la terre (jeunes, investisseurs, etc.).

\section{Méthodologie}

Pour expliquer les phénomènes du morcellement informel et celui de la diversité comportementale, nous avons eu recours à la théorie de North. Pour ce dernier, le rôle des agents est central dans le changement économique et institutionnel, mais il considère que la compréhension de leur comportement nécessite de prendre en compte l'existence de chemins de dépendance historique et institutionnel (Chabaud et al., 2005). Cette notion de "path dependence" souligne le poids des choix effectués dans le passé et celui des institutions politiques sur les décisions présentes. 


\section{Étude de cas}

Une enquête a été réalisée dans le but d'expliquer la diversité des comportements des attributaires et d'établir une typologie. Elle a eu lieu dans le périmètre irrigué de la Mitidja Ouest qui dépend administrativement des communes de Mouzaïa et de Attatba, faisant respectivement partie des wilayates de Blida et de Tipaza (figure 1). Elle s'est déroulée entre mars et juin 2008 dans l'ex-DAS Boudjema Ikhlef (figure 2). La pluviométrie dans cette zone dépasse en moyenne $600 \mathrm{~mm}$ par an et le sol argileux convient à l'arboriculture. Les études de cas ont été menées sur la base d'un guide d'entretien portant sur l'identification des attributaires (qualification et poste occupé durant la période de l'autogestion), les difficultés rencontrées après la réforme de 1987, les conflits et les raisons du partage informel, les investissements réalisés et enfin le mode de faire-valoir.

L'échantillon est composé de 48 cas qui n'ont pas été choisis au départ. Un cas est un attributaire ou un(e) héritier(e), qui représente une EAC unie ou morcelée, ou encore un preneur qui a eu accès à l'EAC par le biais d'un contrat informel. Par ailleurs, la préoccupation fondamentale qui guidait les enquêteurs a été de saisir la diversité des comportements sachant qu'aucune étude antérieure n'a été faite sur le sujet. Ainsi, "la représentativité dont il s'agit ici ne résulte pas de formes de généralisation statistique ou prédictive. Elle est qualitative et inductive, visant à expliquer et à "abstraire " des mécanismes et des processus " (Le Meur, 2002).

\section{Établissement de la typologie comportementale}

$\mathrm{Au}$ fur et mesure du déroulement de l'enquête, de nouveaux cas ont été explorés. Mais, à partir d'un certain seuil, les enquêtes sont devenues répétitives : c'est le phénomène de saturation. "La notion de saturation correspond d'une certaine façon au principe d' ignorance optimale" proposée par Robert Chambers (1991 : 552), qui est une invitation à ne pas chercher l'exhaustivité pour elle-même (idée de toute manière chimérique) mais à se poser la question de savoir jusqu'où l'approfondissement de l'enquête est nécessaire et productif par rapport aux objectifs de celle-ci." (Le Meur, 2002).

Par conséquent, les critères qui ont servi à l'établissement de la typologie de comportement ont été identifiés durant l'enquête. Mais pour atteindre la saturation, il a été inévitable d'aborder des cas présentant des similitudes avec les cas ayant déjà fait l'objet d'une enquête, ce qui a conduit par la suite à réduire les 48 cas étudiés à 10 types de comportement.

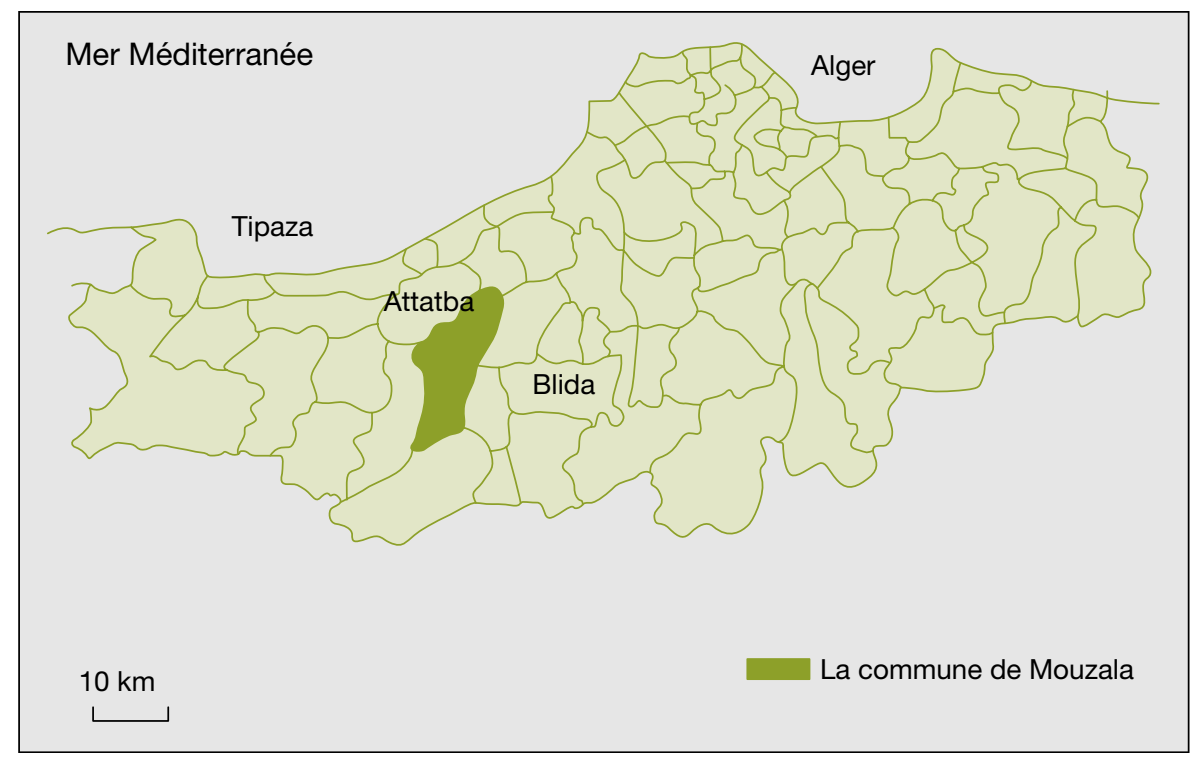

Figure 1. La position géographique de Mouzaia (zone d'étude).

Figure 1. Geographical location of Mouzaïa (study zone).

\section{Résultats}

\section{Analyse de la trajectoire d'évolution de l'EAC 2 située dans l'ex-DAS Boudjema Ikhlef}

Cette analyse cherche à illustrer les difficultés rencontrées par les attributaires et qui ont favorisé le processus du morcellement informel. Cet exemple a été choisi car le partage informel de l'EAC s'est produit deux fois successivement, ce qui montre le caractère évolutif de ce phénomène.

À la réforme de 1987, l'EAC 2 comptait 13 attributaires sur une superficie agricole utile de 58,67 hectares, dont 50,45 hectares de terres nues et 8,22 hectares de vergers (agrumes et pruniers). Par la suite, des conflits ont éclaté entre les membres du collectif de départ à cause de problèmes liés au manque d'eau et à l'échec dans la réalisation d'un forage. Un premier partage informel a eu lieu qui a abouti à la constitution de deux sousgroupes (de 7 et 4 attributaires) et de deux attributaires indépendants. Ce partage a rétabli provisoirement un ordre social mais le problème de l'eau sévissait toujours. Face à cette contrainte, le sousgroupe de sept attributaires décida de louer une partie de l'exploitation et d'emblaver l'autre partie en céréales. Cette stratégie permettait au sous-groupe de rester uni mais avec de faibles niveaux de revenus. En effet, la location d'un hectare de terre nue pour une campagne agricole ne dépasse pas 15000 dinars (soit l'équivalent du salaire minimum garanti mensuel) ; et le reste de la superficie, correspondant à 1,5 hectare par attributaire, procurait un revenu insuffisant avec un rendement moyen de $10 \mathrm{q} / \mathrm{ha}$. Aussi, le sous-groupe des 7 attributaires a-t-il opté pour un deuxième partage informel en trois sous-groupes, constitués respectivement l'un de 5 attributaires et les deux autres d'un attributaire indépendant chacun.

\section{Typologie comportementale}

La typologie de comportement des agriculteurs enquêtés dans les EAC de l'ex-DAS Boudjema Ikhlef a été établie sur la base des critères déterminant le partage du foncier et les arrangements informels entre attributaires et preneurs: réalisation d'investissements 


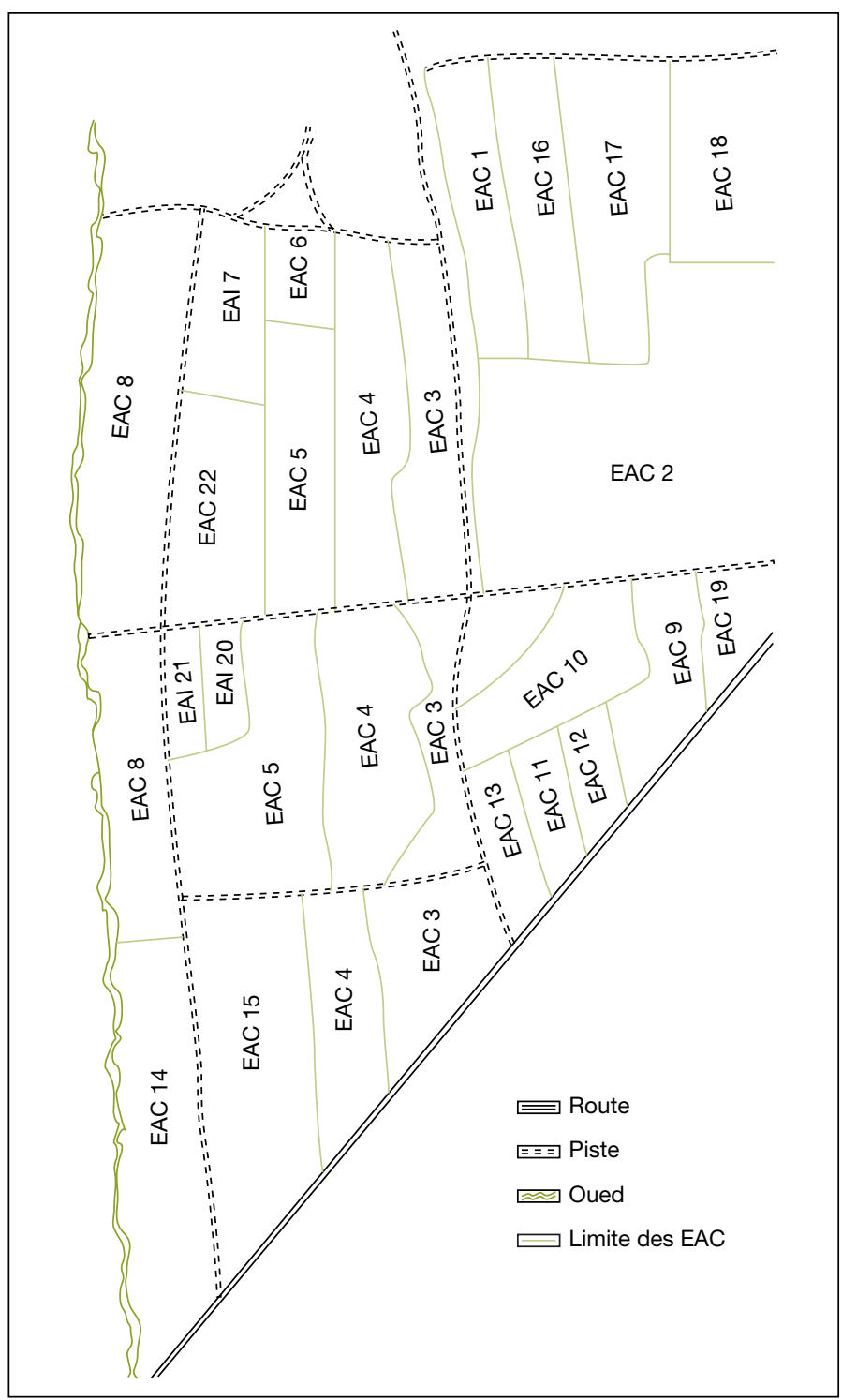

Figure 2. Parcellaire de l'ex-domaine agricole socialiste (DAS) Boudjema lkhlef.

Figure 2. Plot of the former Boudjema Ikhlef Socialist Agricultural Domain.

(puits, plantations), mode de gestion de l'exploitation, location de terres, pluriactivité, etc. Ces critères sont présentés dans une grille d'analyse (tableaux 1 et 2).

Les dix types de comportement identifiés sont présentés ci-après.

Type 1:

comportement d'entrepreneur (forme évolutive) (17 cas)

Ce groupe est composé d'attributaires pratiquant une agriculture dynamique et rentable (vergers irrigués). Ils ont su surmonter les difficultés liées aux problèmes d'eau et de financement, mais aussi les conflits internes, grâce à leur volonté et à leur savoir-faire acquis avant ou après la réforme de 1987. Ce type de comportement a été observé chez les ex-cadres (gestionnaires, comptables, techniciens) des ex-DAS mais aussi quelquefois chez d'anciens ouvriers qui ont progressé en imitant les agriculteurs les plus performants. Ces entrepreneurs optent pour un mode de faire-valoir direct (et parfois pour un mode de faire-valoir mixte, direct et indirect) et restent les gestionnaires de leurs exploitations.

Type 2:

comportement d'entrepreneur

(forme régressive) (4 cas)

Ces attributaires ont été freinés dans leur évolution par des procédures judiciaires (conflits internes) ou des échecs dans la réalisation des investissements (échec d'un forage, plantations souffrant du manque d'eau, etc.). Ces problèmes ont conduit à diverses évolutions: terres cédées en mode de faire-valoir indirect, dispersion des membres du collectif de l'exploitation, recherche d'un revenu dans une autre exploitation agricole (fermage, métayage) ou dans une activité économique non agricole.

Type 3 :

comportement de repli (6 cas)

Ces attributaires ont cédé la gestion de leurs exploitations à des intervenants privés après avoir réalisé des investissements rentables (forages, plantations). Toutefois, ils ont continué d'exercer l'activité agricole comme ouvriers saisonniers dans leur propre exploitation ainsi que dans d'autres exploitations agricoles à temps partiel. Ils étaient de simples exécutants dans les ex-DAS (ouvriers). Finalement, ces attributaires n'ont pas pu assumer le statut d'exploitant agricole et assurer la gestion de l'exploitation.

Type 4 :

comportement de démission (6 cas)

Ces attributaires ont cédé leurs exploitations en mode de faire-valoir indirect (contrats informels à moyen et long termes) sans avoir réalisé d'investissements. Et ils ont opté pour des activités non agricoles. Ce type de comportement regroupe des attributaires âgés, parfois retraités, qui ont exercé durant la période de l'autogestion des postes d'ouvriers ou des fonctions éloignées de l'activité agricole (mécanicien, pointeur, gardien, etc.).

Type 5:

comportement de l'héritière (1 cas)

L'épouse héritière d'une quote-part s'est retrouvée seule face à une responsabilité à laquelle elle n'était pas préparée. Elle a donc opté pour un mode de faire-valoir indirect. Son objectif est de tirer profit de cette parcelle, mais elle n'a ni les moyens ni le savoir-faire pour engager des investissements.

Type 6 : comportement en instance d'identification ( 1 cas)

Après le morcellement informel, un attributaire a cédé sa quote-part en mode de faire-valoir indirect pendant plusieurs années. Par la suite, il a repris la gestion de sa quote-part en plantant une parcelle et en cédant en fermage une autre parcelle. 
Type 7 : comportement du conquérant investisseur ( 6 cas)

Les conquérants investisseurs regroupent aussi bien des attributaires que des intervenants privés, avec ou sans terre. En majorité, ces conquérants n'exercent que l'activité agricole et réalisent des investissements importants (forages, plantations), cherchant des opportunités pour maximiser les profits. Il faut noter que ces conquérants prennent des risques réels du fait que le mode de faire-valoir indirect est interdit par la loi.

Type 8: comportement du conquérant locataire ( 1 cas)

Ce conquérant locataire est un preneur privé qui intervient dans le financement de la campagne agricole (notamment la fertilisation et le traitement) mais reste sous le contrôle du bailleur (attributaire). Le contrat qui relie le preneur au bailleur est informel. Bien qu'un seul cas ait été exploré, nous savons que ces conquérants locataires sont nombreux (attributaires et intervenants privés avec ou sans terre).

Type 9 : comportement du conquérant sous-locataire (5 cas)

Il s'agit de jeunes exploitants sans terre, spécialisés dans les cultures sous serres (poivrons) qui viennent de régions limitrophes (Beni-Tamou, Oued El-Alleug). Ils louent la terre à des intervenants et assurent le financement des serres.

Type 10 : comportement du conquérant opportuniste (1 cas)

Cet acteur économique, non-agriculteur, a accédé au foncier agricole du domaine privé de l'État (terres agricoles appartenant à l'État et qui peuvent être vendues ou transférées à des particuliers) avec l'intention de tirer profit d'une conjoncture instable. Il exploite la faiblesse des

Tableau 1. Critères d'identification des types de comportements des attributaires.

Table 1. Indicators of beneficiary behavioural types.

\begin{tabular}{|c|c|c|c|c|c|c|c|c|}
\hline \multirow{2}{*}{$\begin{array}{l}\text { Acteurs } \\
\text { économiques }\end{array}$} & \multirow[t]{2}{*}{ Critères } & & \multicolumn{6}{|c|}{ Types de comportements } \\
\hline & & & T1 & T2 & T3 & T4 & T5 & T6 \\
\hline \multirow[t]{10}{*}{ Attributaires } & \multirow[t]{3}{*}{ Investissements } & $\begin{array}{l}\text { Investissements } \\
\text { rentables }\end{array}$ & $x$ & & $x$ & & & $x$ \\
\hline & & $\begin{array}{l}\text { Échec des } \\
\text { investissements }\end{array}$ & & $x$ & & & & \\
\hline & & $\begin{array}{l}\text { Absence des } \\
\text { investissements }\end{array}$ & & & & $x$ & $x$ & \\
\hline & \multirow{3}{*}{$\begin{array}{l}\text { Mode de } \\
\text { faire-valoir }\end{array}$} & Direct & & & & & & \\
\hline & & Direct et indirect & $x$ & & & & & $x$ \\
\hline & & Indirect & & $x$ & $x$ & $x$ & $x$ & \\
\hline & \multirow{3}{*}{$\begin{array}{l}\text { Activités } \\
\text { économiques }\end{array}$} & Agricole & $x$ & & & & & \\
\hline & & $\begin{array}{l}\text { Agricole et non } \\
\text { agricole }\end{array}$ & & $x$ & $x$ & $x$ & & $x$ \\
\hline & & Non agricole & & & & & $x$ & \\
\hline & Autres & $\begin{array}{l}\text { Retour à } \\
\text { l'exploitation }\end{array}$ & & & & & & $x$ \\
\hline
\end{tabular}

attributaires en difficulté pour accéder au foncier et spécule ensuite sur un marché foncier informel.

\section{Discussion}

L'analyse du processus de morcellement informel dans les EAC de l'ex-domaine agricole socialiste Boudjema Ikhlef, a permis d'identifier une grande diversité de comportements qui s'expliquent par l'existence de chemins de dépendance historique. En effet, comme nous en avons fait l'hypothèse, les dynamiques mises en évidence sont en relation étroite avec le passé des attributaires pendant la période de l'autogestion (1963-1987).

Les attributaires qui sont parvenus à surmonter les difficultés (problèmes d'eau, financements, conflits) (type 1) sont principalement les ex-cadres des DAS ayant acquis dans le passé des connaissances et une expérience en matière de gestion et qui ont notamment bénéficié de stages de formation en comptabilité et en agri-

Tableau 2. Critères d'identification des types de comportements des intervenants.

Table 2. Indicators of tenant behavioural types.

\begin{tabular}{|c|c|c|c|c|c|c|}
\hline \multirow[t]{2}{*}{ Acteurs économiques } & \multirow[t]{2}{*}{ Critères } & & \multicolumn{4}{|c|}{ Types de comportement } \\
\hline & & & T7 & T8 & T9 & T10 \\
\hline \multirow{5}{*}{$\begin{array}{l}\text { Intervenants } \\
\text { (preneurs) }\end{array}$} & Investissements & Investissements rentables & $x$ & & & \\
\hline & & Absence d'investissements & & $x$ & $x$ & $x$ \\
\hline & Types de location & Location à court terme & & $x$ & & \\
\hline & & Location à moyen et long termes & $x$ & & & $x$ \\
\hline & & Sous-location à court terme & & & $x$ & \\
\hline
\end{tabular}


culture. Ce sont aussi d'anciens ouvriers qui ont pu investir et faire des progrès par apprentissage (imitation). À l'opposé, ceux qui ont opté pour un mode de faire-valoir indirect ou qui ont tout simplement abandonné leur exploitation étaient des ouvriers agricoles ou exerçaient des activités non agricoles (gardien, chauffeur, pointeur, etc.). Ces attributaires ont eu des comportements de repli (type 3) ou de démission (type 4). Cela a permis l'accès au foncier à plusieurs types d'intervenants conquérants qui se sont substitués aux attributaires (types 7, 8 et 9). Ces nouveaux agriculteurs, pourvus de ressources financières et/ou de technicité, représentent $25 \%$ de notre échantillon. Ils pratiquent une agriculture spécialisée (vergers, serres) à haute valeur ajoutée sur un foncier précaire (contrats informels illégaux).

Il faut noter que des phénomènes de chemin de dépendance historique ont été également observés dans l'analyse de la transition agricole des ex-pays socialistes de l'Europe de l'Est. En Roumanie par exemple, où la décollectivisation a été massive (loi 18/91), on observe le maintien de grandes sociétés agricoles regroupant des ménages ruraux et issues des anciennes coopératives agricoles (Amblard et Colin, 2006). Ces initiatives ont été incitées et soutenues par les politiques agricoles avec l'objectif d'éviter la fragmentation de l'appareil de production.

En Algérie, au contraire, le mode de production collectif a été une obligation institutionnelle, dans un contexte de désengagement de l'État.

Par ailleurs, les pouvoirs publics continuent de refuser de reconnaitre le morcellement informel des EAC par crainte d'aboutir à la généralisation de petites exploitations agricoles peu performantes et de favoriser la spéculation foncière.

Cette appréhension est objectivement non justifiée puisque nous avons observé un réel dynamisme agraire dans la zone étudiée, alimenté par des attributaires entrepreneurs actifs (type 1) et les preneurs de terre conquérants (types 7 , 8 , 9), soit $60 \%$ de notre échantillon. De plus, nous n'avons relevé qu'un seul cas de spéculation sur 48 (type 10).

Enfin, rappelons que le morcellement de la terre ne porte pas obligatoirement préjudice à la modernisation de l'agriculture (Jouve, 2001). L'expérience de l'Albanie, dont la réforme agraire égalitaire a provoqué un morcellement extrême de la terre agricole, montre que les paysans "extrêmement désillusionnés par le système de l'agriculture collective, se sont vite adaptés à leur nouvelle situation de 'fermage privé, même si la superficie de leur ferme reste limitée, variant de 0,6 à 2,5 ha par famille... et qu'après dix ans d'expérience (1990-2000), on observe les signes de l'orientation de l'agriculture albanaise vers une agriculture de type familial" (Civici, 2001).

Le point le plus problématique de ce phénomène de morcellement tient à son caractère informel et à l'insécurité foncière qu'il alimente. L'autorisation des transactions foncières permettrait de sortir de l'informel. Dans ce sens, l'expérience chinoise est intéressante qui, sans remettre en cause la propriété étatique de la terre, a instauré un système contractuel (le "système de responsabilité du ménage ") autorisant le recours au bail pour les terres agricoles (Jianping et Jian, 2008).

\section{Conclusion}

L'objectif de cet article est d'expliquer les phénomènes du morcellement informel des exploitations agricoles collectives en Algérie ainsi que la diversité comportementale des attributaires. Comme dans les autres pays ayant adopté le modèle agricole socialiste, les attributaires ont été en majorité des ouvriers et donc de simples exécutants dans les domaines agricoles socialistes. Le passage du statut d'ouvrier à celui d'exploitant agricole et de gestionnaire a été vécu comme une rupture, au moment de la transition économique introduite par la réforme de 1987.

En outre, ces attributaires ne sont pas parvenus à évoluer dans une forme de production collective. Des conflits ont éclaté entre les membres des EAC et ont mené vers une décollectivisation quasi générale. Ainsi, le passage brutal du domaine agricole socialiste géré directement par l'Etat à une forme de production collective imposée par la réglementation n'a pas donné les résultats escomptés.

Le morcellement informel s'est présenté alors comme l'ultime solution pour rétablir de l'ordre. L'éclatement du pouvoir de décision économique et technique inhérent à ce morcellement a mis en évidence une grande diversité des comportements des attributaires et des trajectoires d'exploitations (10 types).

En conclusion, la généralisation du morcellement informel des exploitations agricoles collectives et de la diversité comportementale des attributaires peut se comprendre comme la conséquence de l'inadaptation des règles formelles imposées par la loi 87-19 et montre les limites d'une conception qui n'a pas tenu compte des aspects sociaux et culturels dans la gestion du changement économique et institutionnel. Dans ce sens, les expériences de transition agricole des ex-pays socialistes de l'Europe de l'Est, enchâssées dans leurs contextes socioéconomiques et culturels respectifs, peuvent donner des enseignements mais ne pourraient pas servir de modèle à expérimenter en Algérie sans risque d'échec.

\section{Références}

Amblard L, Colin JP. Dimension organisationnelle et pratiques contractuelles foncières: les sociétés agricoles en Roumanie. Economie Rurale 2006 ; 293 : 55-71.

Baci L. Les réformes agraires en Algérie. In: Jouve AM et Bouderbala N, eds. Politiques foncières et aménagement des structures agricoles dans les pays méditerranéens. Cahiers Options méditerranéennes 1999; 36 : 285-91.

Bedrani S. L'agriculture algérienne depuis 1966. Alger: OPU, 1981.

Chabaud D, Parthenay C, Perez Y. L'évolution de I'analyse northienne des institutions: la prise en compte des idéologies. Revue Economique $2005 ; 56: 691-703$.

Civici A. Évolution des politiques foncières et dynamique des espaces ruraux en Albanie. In: Jouve AM, ed. Terres méditerranéennes. Le morcellement, richesse ou danger?. Paris : KarthalaCiheam, 2001.

Imache A. Construction de la demande en eau agricole au niveau régional en intégrant le comportement des agriculteurs: application aux exploitations agricoles collectives de la Mitidjaouest (Algérie). Thèse de doctorat. Paris: AgroParisTech, 2008. www.eau-sirma.net/les_formations/les-theses/instruments-d-analyse-des-performances/construction de la demande d eau agricole_au niveau_regional_en_integrant_le_ comportement_des_agriculteurs_application aux_exploitations_agricoles_collectives_de_la_mitidja_ouest_algerie_these_de_a_imache

Jianping $Y$, Jian $W$. Les réformes foncières en république populaire de Chine. Etudes Foncières $2008 ; 132$ : 36-41.

Jouve AM. Transitions foncières dans les Balkans: Roumanie, Albanie, Grèce. Options méditerranéennes, Série A (82). Montpellier: Ciheam, 2009.

Jouve AM. Terres méditerranéennes. Le morcellement, richesse ou danger. Paris: KarthalaCiheam, 2001.

Kuper M, Errahj M, Faysse N, Caron P, Djebbara $M$, Kemmoun H. Autonomie et dépendance des irrigants en grande hydraulique: observations de I'action organisée au Maroc et en Algérie. Nature Sciences Sociétés 2009 ; 17 : 248-56.

Le Coz J. L'Algérie. Décennie 1980 : Les étapes de la désocialisation. Espace rural 1991; (24) : 94 p.

Le Meur PY. Approche qualitative de la question foncière. Note méthodologique. Montpellier: IRD REFO, 2002. www.foncier-developpement. org/analyses-et-debats 